\title{
A novel combined conjugate vaccine: Enhanced immunogenicity of bFGF with CRM197 as a carrier protein
}

\author{
HAI-LONG ZHANG ${ }^{1 *}$, CHUANG YUAN $^{1 *}$, DONG-MEI ZHANG ${ }^{1}$, HUA-SHAN SHI $^{1}$, \\ MENG LI ${ }^{1}$, ZI-CHAO LUO ${ }^{2}$, YANG WAN ${ }^{1}$, LIAN LU ${ }^{1}$, SHUN-TAO LUO $^{1}$ and LI YANG ${ }^{1}$ \\ ${ }^{1}$ State Key Laboratory of Biotherapy and Cancer Center, West China Hospital, West China Medical School, \\ Sichuan University; ${ }^{2}$ School of Life Science, Sichuan University, Chengdu, Sichuan, P.R. China
}

Received March 23, 2011; Accepted June 27, 2011

DOI: $10.3892 / \mathrm{mmr} .2011 .521$

\begin{abstract}
Tumor growth is partly dependent on tumor-associated angiogenesis, which is regulated by angiogenic growth factors. As the first angiogenic growth factor to be identified, basic fibroblast growth factor (bFGF) plays a major role in angiogensis and tumor growth and has been an effective target for anti-tumor therapy. However, due to its low immunogenicity, injection with bFGF alone cannot stimulate the body to produce a strong immune response. In this study, we investigated the role of CF (containing bFGF and CRM197) assisted by $\mathrm{CpG}$ and alum in enhancing antigen-specific immune response and suppressing the growth of murine colon carcinoma. The results revealed that compared to bFGF, CF could not stimulate NIH-3T3 fibroblast proliferation even at a concentration of $10 \mu \mathrm{g} / \mathrm{ml}$ in vitro. In vivo, the CF-CpG-alum produced a stronger antigen-specific immune response and inhibited tumor growth. The anti-tumor activity was associated with generating antigen-specific antibody, suppressing angiogenesis, promoting the apoptosis of tumor cells and inducing the mixed Th1 and Th2 responses. This indicates that CRM197 may be an innovative intramolecular adjuvant and provides a rational preservation for mouse CT26 colon carcinoma.
\end{abstract}

Correspondence to: Professor Li Yang, State Key Laboratory of Biotherapy, West China Hospital, Sichuan University, Keyuan Road 4, Chengdu, Sichuan 610041, P.R. China

E-mail: yl.tracy73@gmail.com

*Contributed equally

Abbreviations: bFGF, basic fibroblast growth factor; CRM197, nontoxic mutant of diphtheria toxin; NIH-3T3, mouse embryonic fibroblast cell; CT26, mouse colon carcinoma cell; VEGF, vascular endothelial growth factor; TGF- $\alpha / \beta$, transforming growth factor- $\alpha / \beta$; TNF- $\alpha$, tumor necrosis factor- $\alpha$; PVDF, polyvinylidene difluoride; MVD, microvessel density; PBS, phosphate buffered saline

Key words: tumor, angiogenesis, basic fibroblast growth factor, CRM197, CpG

\section{Introduction}

Angiogenesis is the process of new vascular network formation from existing vessels (1). This process not only plays a key role in various physiological activities but also occurs in pathological conditions, such as embryonic development, wound healing, rheumatoid arthritis and diabetic retinopathy $(1,2)$. Accordingly, tumor growth is partly dependent on angiogenesis or neovascularisation $(3,4)$. Antiangiogenesis may therefore be an effective strategy of antitumor treatment through inhibiting tumor growth (5-8).

Tumor angiogenesis is a multi-step process, which involves endothelial and smooth muscle cell proliferation and migration, formation of new capillaries, and extracelluar matrix turnover $(9,10)$. Certain studies have indicated that a host of diffusible factors produced by tumor cells mediate these processes $(11,12)$. Since then, an increasing number of angiogenic cytokines have been identified, including basic fibroblast growth factor (bFGF), the vascular endothelial growth factor (VEGF) family, angiopoietins, interleukin-8, transforming growth factor- $\alpha$ and $-\beta$ (TGF- $\alpha$ and $-\beta$ ) and tumor necrosis factor- $\alpha$ (TNF- $\alpha)(13-15)$.

Basic fibroblast growth factor (bFGF), one of the first angiogenic growth factors to be identified, is a single-chain soluble heparin-binding polypeptide $(16,17)$. It induces the migration and proliferation of endothelial cells and smooth muscle cells and the formation of vascular tubes and networks. In addition, evidence that bFGF plays an important role in angiogenesis has been provided by numerous experiments (18-20). It interacts with signaling FGFRs, the extracellular matrix and certain free molecules, and influences the initial period of angiogenesis and tumor growth $(15,21)$. Based on these characteristics, bFGF could be used as a target of tumor therapy. However, due to its short half-life in vivo and low immunogenicity, the biological effect of bFGF is limited and it cannot stimulate the body to produce a strong immune response alone (22). Therefore, a study was carried out targeting a specific immune response to the bFGF molecule for the endogenous control of tumor growth (23).

CRM197 is a nontoxic mutant of diphtheria toxin and is immunologically indistinguishable from the native molecule. CRM197 is not only capable of inhibiting ovarian cancer as a specific inhibitor of HB-EGF $(24,25)$, but also of enhancing 
immunogenicity with capsular polysaccharides (PSs) as a carrier protein to prevent infection (26). As a carrier protein, CRM197 induces a rapid increase in Th1- and Th2-secreting $\mathrm{T}$ cells, which produce heterogeneous cytokines to activate B cells and regulate the quantity of PS antibody $(27,28)$.

$\mathrm{CpG}$ is a type of bacterial DNA or synthetic oligodeoxynucleotide containing immunostimulatory $\mathrm{CpG}$ motifs. $\mathrm{CpG}$ DNA can induce the majority of $(>95 \%)$ B cells to proliferate and predominantly trigger mixed $\operatorname{IgG} 1 / \operatorname{IgG} 2 a$ secretion (29). Moreover, CpG DNA also directly activates monocytes, macrophages and dendritic cells to secrete a host of cytokines. These cytokines stimulate NK cells to secrete IFN- $\gamma$, which promotes B cell activation and Ig secretion (30). Overall, $\mathrm{CpG}$ DNA modulates immune functions by activating lymphocytes, and by modulating Th1/Th2 balance (31).

In this study, we describe that the fusion protein, $\mathrm{CF}$, which conjugates bFGF with CRM197, together with CpG and alum as an adjuvant, enhances the immunogenicity of bFGF and inhibits tumor angiogenesis and growth.

\section{Materials and methods}

Cell and cell cultures. Murine colon carcinoma cell line (CT26), purchased from the American Type Culture Collection (ATCC), was cultured in RM1640 medium (Gibco BRL, Grand Island, NY, USA) supplemented with $10 \%$ FBS and $100 \mu \mathrm{g} / \mathrm{ml}$ of amikacin and maintained in a humidified chamber at $37^{\circ} \mathrm{C}$ in a $5 \% \mathrm{CO}_{2}$ atmosphere.

Construction of recombinant plasmid. According to the cDNA sequences of CRM197 and hbFGF (28bp-438bp) in GenBank, we designed the corresponding primers that contain the appropriate restriction sites (CRM197, 5'-GCTCTAGAAATAATTT TGTTTAACTTTAAGAAGG-3' and 5'-GGAATTCGCTTTT GATTTCAAAAAACAGCGAC-3'; hbFGF, 5'-GGAATTCCC GCCTTGCCCGAGGATGGC-3' and 5'-CCCAAGCTTATC AGCTCTTAGCAGACATTGGAAG-3'). Then, the cDNA fragments coding the CRM197 or hbFGF genes were amplified by PCR and subsequently subcloned into the pET-32a(+) vector (Novagen). The expressed recombinant protein, $\mathrm{CF}$, consisted of CRM197 and hbFGF (10aa-155aa) (Fig. 1).

Protein expression and purification. Escherichia coli cells [BL21 (DE3) strain] harboring pET32a-CF were grown in 41 of $\mathrm{LB}$ medium at $37^{\circ} \mathrm{C}$ with the selection marker, ampicillin $(100 \mu \mathrm{g} / \mathrm{ml})$. When the OD600 of the culture reached 0.6-0.8, the isopropyl-b-D-thiogalactopyranoside was added into it with the final concentration of $0.1 \mathrm{mM}$ and the culture was maintained at $30^{\circ} \mathrm{C}$ for $16 \mathrm{~h}$. Approximately $20 \mathrm{~g}$ (wet weight) of cells were harvested by centrifugation at $4500 \mathrm{xg}$ and $4^{\circ} \mathrm{C}$ for $30 \mathrm{~min}$. After being washed with ice-cold buffer $(20 \mathrm{mM}$ Tris-HCl, $150 \mathrm{mM} \mathrm{NaCl}, \mathrm{pH} 8.0$ ), the cells were resuspended in $200 \mathrm{ml}$ lysis buffer $(20 \mathrm{mM}$ Tris- $\mathrm{HCl}, \mathrm{pH} 8.0,150 \mathrm{mM}$ $\mathrm{NaCl}, 10 \mathrm{mM}$ EDTA, $\mathrm{pH}$ 8.0). The suspension was broken with a high-pressure homogenizer at a pressure of $600 \mathrm{Bar}$ and then centrifuged at $70,000 \mathrm{x} \mathrm{g}$ for $30 \mathrm{~min}$ at $4{ }^{\circ} \mathrm{C}$. The inclusion bodies were obtained and then washed twice with $100 \mathrm{ml}$ of buffer A (20 mM Tris-HCl, pH 8.0, 4 M urea, $20 \mathrm{mM} \beta$-ME). Then, the inclusion bodies were solubilized with $50 \mathrm{ml}$ of buffer B (20 mM Tris- $\mathrm{HCl}, \mathrm{pH} 8.0,8 \mathrm{M}$ urea,

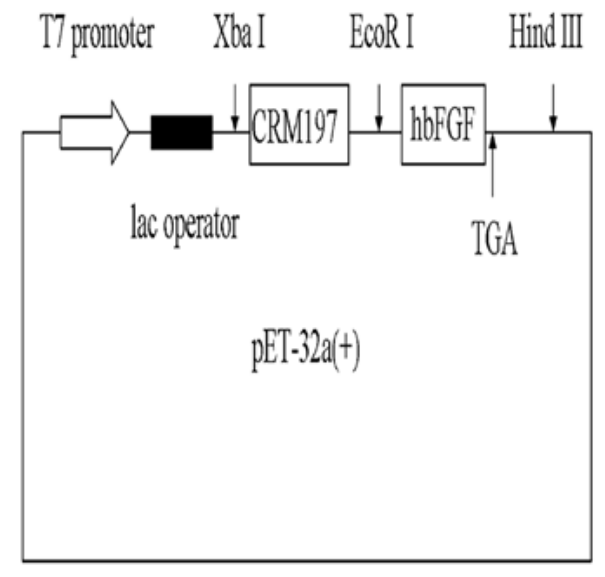

Figure 1. Construction of a vector for the expression of CF. The cDNA fragements coding the CRM197 or hbFGF genes were amplified by PCR and cloned into the pET-32a(+) vector. The fusion protein consisted of CRM197 and hbFGF (10aa-155aa).

$20 \mathrm{mM} \beta-\mathrm{ME}$ ) and subsequently centrifuged at $70,000 \mathrm{x}$ g for $30 \mathrm{~min}$ at $4^{\circ} \mathrm{C}$. The supernatant was slowly dropped into 11 of refolding buffer, and then stirred continuously for $48 \mathrm{~h}$. The refolding sample was concentrated by ultrafiltration and the buffer was transferred to buffer $\mathrm{C}$ (20 mM Tris- $\mathrm{HCl}, \mathrm{pH}$ 8.0). The refolding solution was loaded onto an SP chelating sepharose (GE Healthcare Bio-science) column equilibrated with binding buffer ( $20 \mathrm{mM}$ Tris- $\mathrm{HCl}, \mathrm{pH}$ 8.0). The column was subsequently washed with the binding buffer and further washed with buffer D $(20 \mathrm{mM}$ Tris- $\mathrm{HCl}, 100 \mathrm{mM} \mathrm{NaCl}$, $\mathrm{pH}$ 8.0). The absorbed proteins were eluted with the buffer E, in which the $\mathrm{NaCl}$ concentration was $300 \mathrm{mM}$. Eventually, the protein solution was dialyzed with $10 \mathrm{mM}$ PBS.

Western blot analysis. Western blot analysis was used to confirm the characterization of CF. To start with, the purified $\mathrm{CF}$ was electrophoresed in 10\% SDS-PAGE and transferred onto a polyvinylidene difluoride (PVDF) membrane (BioRad, Richmond, CA, USA). After being washed with PBST, the membrane was blocked with 5\% skimmed milk powder at $4^{\circ} \mathrm{C}$ overnight. Then the blots were incubated with bFGFimmunized mouse serum or CRM197-immunized rabbit serum plus the secondary antibody, HRP-protein A (1:3000, Cabliochem, Darmstadt, Germany). Five washes (5 min per wash) were carried out after each incubation step. The protein bands were visualized via an enhanced chemiluminescence detection system (Pierce, Rockford, IL, USA).

Animal experiments. Female 8-week-old Balb/c mice were purchased from the West China Experimental Animal Center and housed at standard conditions: $22 \pm 2^{\circ} \mathrm{C}$ temperature, $55 \pm 5 \%$ relative humidity and pathogen-free cages. Mice were accommodated for 1 week prior to immunization.

Groups of mice (n=5) were immunized with CpG $(20 \mu \mathrm{g} /$ mouse) and alum mixed with equal volumes of various proteins: i) CRM197 (20 $\mu \mathrm{g}$ /mouse), ii) bFGF (20 $\mu \mathrm{g} /$ mouse), iii) CF (25 $\mu \mathrm{g} /$ mouse) in PBS, or iv) with PBS alone. Mice received a subcutaneous injection $(100 \mu \mathrm{l})$ into both fold inguen once every two weeks (3 times in total), and the day of the first immunization was considered as 
day 0 . Each mouse was challenged subcutaneously with $3 \times 10^{5}$ CT26 cells in the right flank on day 36 . Following this, the tumors were palpable on day 44 and the tumor dimensions were measured with a vernier caliper every 3 days. The tumor volume was calculated by the standard formula (widh $\mathrm{x}$ width $\mathrm{x}$ length $\mathrm{x}$ 0.52). On day 62, the mice were sacrificed. Their organs (heart, liver, spleen, lung and kidney) and tumors were removed and fixed in $4 \%$ paraformaldehyde in PBS for histological analysis. All experiments involving mice were approved by the institute's Animal Care and Use Committee.

Detection of anti-bFGF antibodies and IgG subclasses in blood. Blood samples from immunized mice were obtained once every two weeks during the immunization period and stored at $-80^{\circ} \mathrm{C}$. The anti-bFGF antibodies were measured in enzyme-linked immunosorbent assay (ELISA). Microwell plates (Dynatech Laboratories, Chantilly, VA, USA) were coated with $100 \mu \mathrm{l}$ of $0.5 \mu \mathrm{g}$ bFGF in $50 \mathrm{mM}$ carbonate/ bicarbonate buffer $(\mathrm{pH}=9.6)$ at $4^{\circ} \mathrm{C}$ overnight. The coated plates were washed three times with PBS-Tween wash buffer and then incubated with a $5 \%$ solution of non-fat dried milk in PBS-Tween for $1 \mathrm{~h}$ at $37^{\circ} \mathrm{C}$. After being washed five times with PBS-Tween, the plates were coated with $100 \mu 1$ of plasma (10-time dilution): 1:10,1:100,1:1,000 and 1:10,000 for $1 \mathrm{~h}$ at $37^{\circ} \mathrm{C}$. After washing the plates five times with PBS-Tween, the assay was performed using peroxidase-conjugated affinipure goat anti-mouse IgG (ZSGB-BIO, Beijing, China) incubated for $1 \mathrm{~h}$ at $37^{\circ} \mathrm{C}$. After five washes with PBS-Tween, the substrate was developed with 3, 3, 5, 5-tetramethylbenzadine (Sigma-Aldrich). The colorimetric reaction was stopped with $0.5 \mathrm{M} \mathrm{H}_{2} \mathrm{SO}_{4} 25$ min later. The absorbances were read by an ELISA plate reader (Thermo LabSystems, Franklin, MA, USA) in $450 \mathrm{~nm}$.

As mentioned above, the $\operatorname{IgG}$ subclasses were identified using HRP-conjugated anti-IgG1, anti-IgG2a and anti-IgG2b as secondary antibodies.

Histological analysis. Primary tumors were fixed in $4 \%$ paraformaldehyde in PBS, embedded in paraffin and cut into $4-\mu \mathrm{m}$ sections. The apoptotic cells within the tumor sections were evaluated by dead-end colorimetric terminal deoxynucleotidyl transferase-mediated dUTP Nick-End labeling (TUNEL) System (Promega, Madison, WI, USA) following the manufacturer's protocol. Apoptotic index was determined by counting the number of apoptotic cells and dividing by the total number of cells in the field (5 highpower fields/slide).

The quantification of microvessel density (MVD) was assessed by the method of Weidner et al (32). The procedure of immunostaining for CD31 has previously been described in detail. The following antibodies and reagents were used: goat anti-mouse CD31 mAb (1:200, Santa Cruz Biotechnology, CA, USA), biotinylated polyclonal rabbit anti-goat (1:100, Santa Cruz Biotechnology), ABC Kit (Vector Laboratories, Peterborough, UK) and DAB substrate kit (ZSJQ-BIO, Beijing, China).

Toxicity observation. Drug toxicity indices including weight loss, ruffled fur, diarrhea, anorexia, skin ulcerations and toxic death were closely observed after the first immunization. After being fixed in $4 \%$ paraformaldehyde in PBS for more than $24 \mathrm{~h}$, the tissues of heart, liver, spleen, lung, and kidney were embedded in paraffin and sliced. Sections were stained with hematoxylin and eosin and observed using the double blind method.

Statistical analysis. SPSS 16.0 was used for statistical analysis. The statistical analysis in each experiment was performed using one-way analysis of variance or the unpaired Student's t-test. The difference was regarded as significant if $\mathrm{P}<0.05$.

\section{Results}

Expression and purification of the fusion protein. After transformation of the BL21 (DE3) bacterium, the recombined plasmid was induced with IPTG and expressed the fusion protein as an inclusion body $(75 \mathrm{kDa})$. The fusion protein, which had been solubilized with a buffer $(20 \mathrm{mM}$ Tris- $\mathrm{HCl}$, $\mathrm{pH}$ 8.0, $8 \mathrm{M}$ urea, $20 \mathrm{mM} \beta$-ME) was refolded and purified with an SP chelating sepharose column (Fig. 2A). Meanwhile, the fusion protein was authenticated with Western blotting (Fig. 2B). Moreover, the proliferative bioactivity of CF was measured in NIH-3T3 cells. CF could not stimulate NIH-3T3 fibroblast proliferation even at a concentration of $10 \mu \mathrm{g} / \mathrm{ml}$ (Fig. 2C).

Detection of anti-bFGF antibodies and IgG subclasses in blood. The mice were immunized with CRM197-CpG-alum, bFGF-CpG-alum, CF-CpG-alum and PBS on days 0, 14 and 28. The blood of the mice was obtained on days 7, 21, 35 and 49 , and the sera were used to analyze anti-bFGF antibodies by ELISA. Mice immunized with CRM197-CpG-alum and PBS did not dispay immunoreactivity to bFGF. However, the antibody did react against bFGF from days 7 to 49 in the mice from the other groups. The $\mathrm{CF}-\mathrm{CpG}$-alum group displayed a stronger immune response to bFGF from the first week $(\mathrm{P}<0.05)$ (Fig. 3A). We then analyzed the positive proportion of immunoreactivity to bFGF in each group. Immunoreactivity to bFGF was exhibited by $40 \%$ of mice immunized with CF-CpG-alum in the first week, whereas it was not observed in the bFGF-CpG-alum group. The latter achieved $20 \%$ positive proportion by the third week and $100 \%$ positive proportion by the seventh week, although the CF-CpG-alum group achieved $100 \%$ by the third week, with a lower bFGF content compared to the bFGF-CpG-alum group (Fig. 3B).

Finally, IgG subclasses in the blood were analyzed in the fifth week. The results showed that $\mathrm{CF}-\mathrm{CpG}$-alum could observably enhance the secretion of $\operatorname{IgG} 1$ and $\operatorname{IgG} 2 \mathrm{a}$ in contrast to bFGF-CpG-alum (Fig. 3C).

Anti-tumor effect of CF-CpG-alum in vivo. A murine colon carcinoma model was established to examine the anti-tumor efficacy of CF-CpG-alum in vivo. Mice were challenged with CT26 cells following the final immunization with CRM197-CpG-alum, bFGF-CpG-alum, CF-CpG-alum or PBS. Alterations in tumor growth were monitored every 3 days (Fig. 4A). On day 26 after the CT26 cells were challenged, the 
A

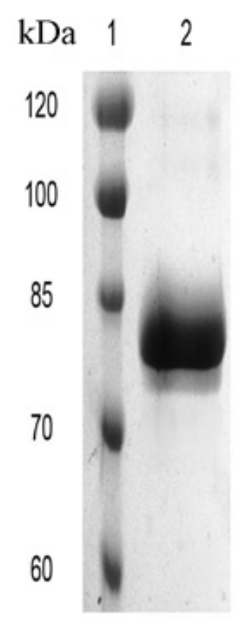

B

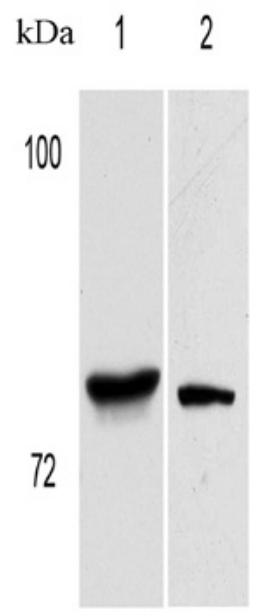

C

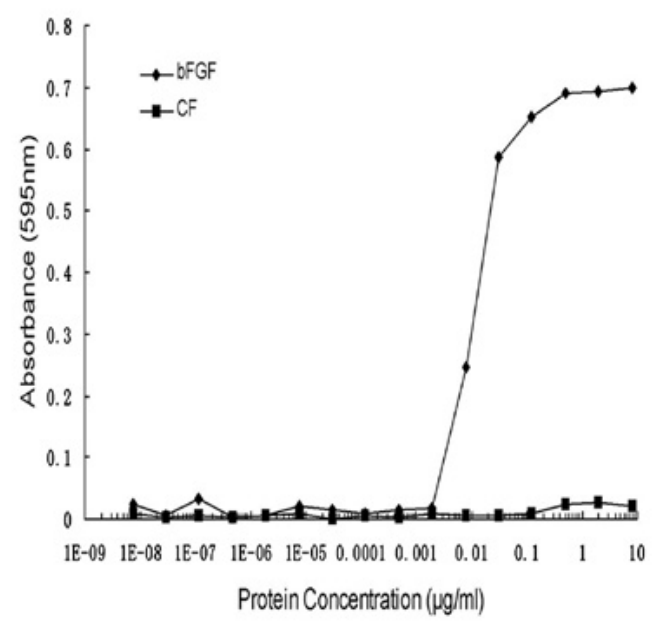

Figure 2. Expression, purification and verification of recombinant protein (CF). (A) SDS-PAGE analysis of the purification of CF expressed as a single band of $75 \mathrm{kDa}$ (lane 2) compared with the marker (lane 1). (B) Western blot analysis of the characterization of CF using anti-CRM197 (lane 1) and anti-bFGF (lane 2). (C) Bioassay of CF compared with bFGF.

A

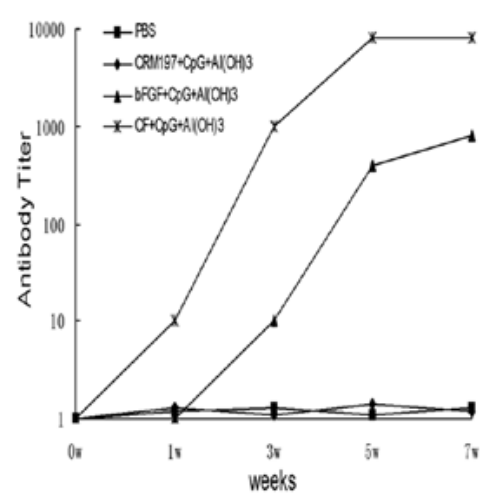

B

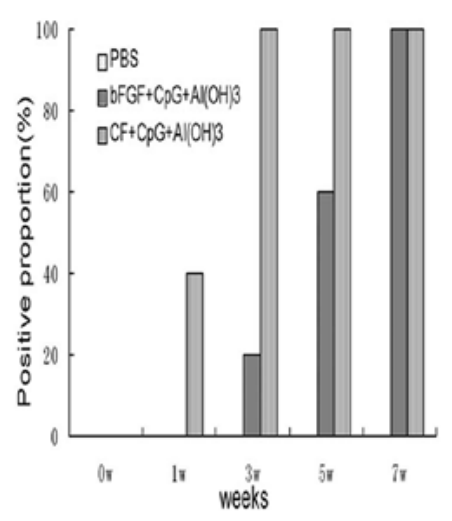

C

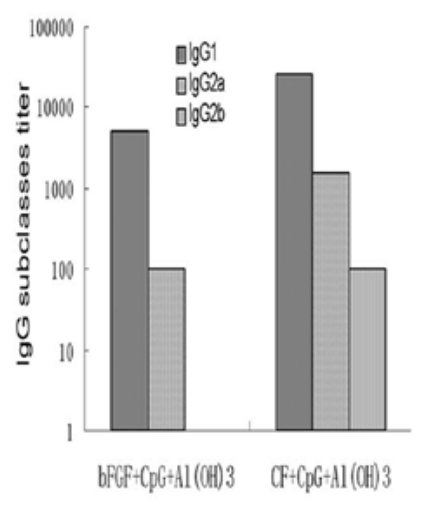

Figure 3. Detection of anti-bFGF antibodies, positive proportion and IgG subclasses. The mice were immunized with corresponding drugs on days 0 , 14, and 28. The blood was subsequently obtained on days 7,21, 35 and 49. Then, the sera were used to analyze (A) the anti-bFGF antibodies, (B) positive proportion and (C) IgG subclasses by ELISA.

mice were sacrificed and the tumor weights were measured (Fig. 4B). The CF-CpG-alum group exhibited effective inhibition of tumor growth, compared to the other groups $(\mathrm{P}<0.01)$. Furthermore, we observed that immunization with $\mathrm{CF}-\mathrm{CpG}-$ alum resulted in average tumor weight reductions of $82.5 \%$, 77.7\% and $71.4 \%$ compared to PBS, CRM197-CpG-alum and bFGF-CpG-alum, respectively $(\mathrm{P}<0.01)$. These results indicate that $\mathrm{CF}-\mathrm{CpG}$-alum can effectively suppress tumor growth.

Increase of apoptosis and inhibition of tumor-induced angiogenesis in vivo. In order to estimate the apoptosis in tumor tissues, the tumor sections were stained with TUNEL reagent (Fig. 5A). Marked necrosis was observed in the CF-CpGalum group (data not shown). Meanwhile, substantially more apoptotic cells were observed in the tumor sections of mice immunized with CF-CpG-alum than with PBS, CPM197CpG-alum or bFGF-CpG-alum. The apoptotic index also revealed that the CF-CpG-alum displayed the highest indices among all the groups (Fig. 5B). The MVDs of tumor sections were analyzed to determine whether the increased apoptosis was associated with anti-angiogenesis in mice immunized with $\mathrm{CF}-\mathrm{CpG}$-alum. Each tumor section was stained with anti-CD31 antibody (Fig. 6A). Tumors of the control groups (including PBS, CRM197-CpG-alum and bFGF-CpG-alum groups) exhibited larger microvessel counts than those of the CF-CpG-alum group (Fig. 6B). These results indicate that the decreased tumor volumes of the CF-CpG-alum group may be caused by increased apoptosis, which was associated with antiangiogenesis.

Toxicity. In order to evaluate the potential side-effects of the drugs, the toxicity assessment was performed. No marked differences were observed in the gross measures among the groups (data not shown). Moreover, no pathological changes were detected in the heart, liver, spleen, lung, or kidney (data not shown). 

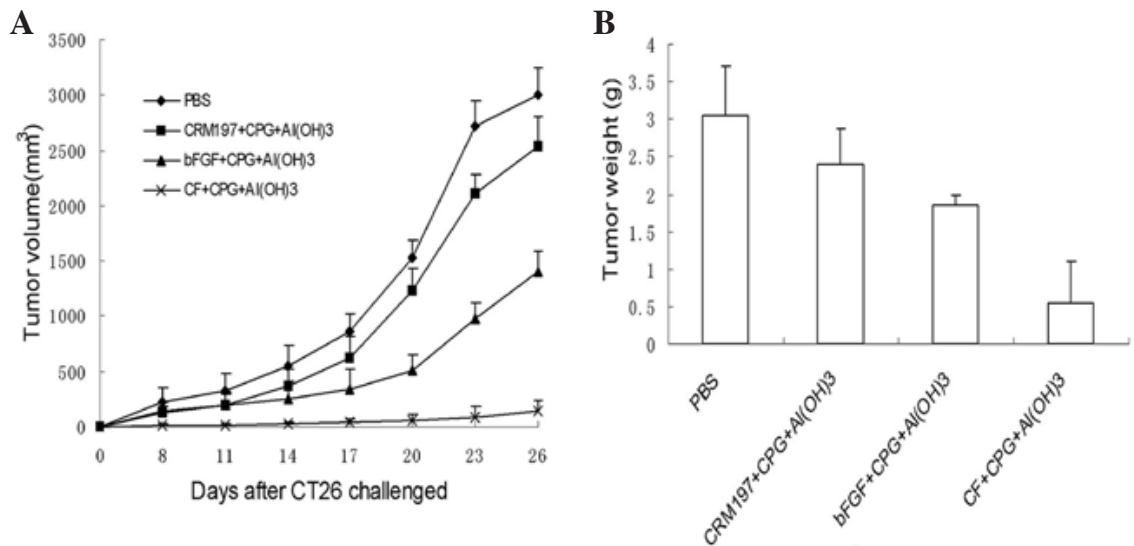

Figure 4. Anti-tumor efficacy of CRM197-CpG-alum, bFGF-CpG-alum and CF-CpG-alum. The mice immunized with corresponding complexes were challenged with $3 \times 10^{5}$ CT26 cells. (A) Tumor sizes on each mouse were monitored every 3 days and (B) tumor weights were measured.
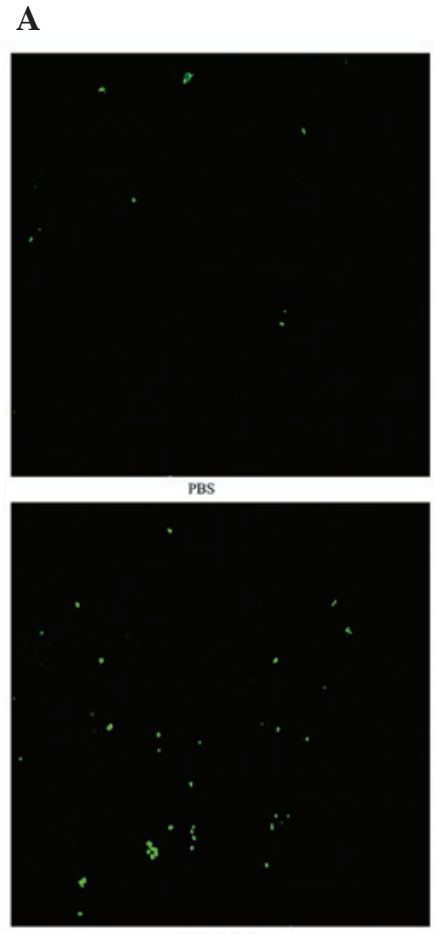

bFGF-CPG-alum
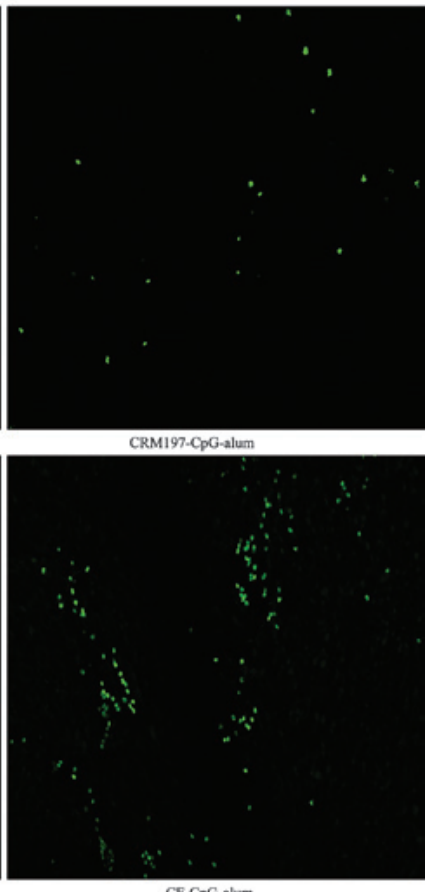

B

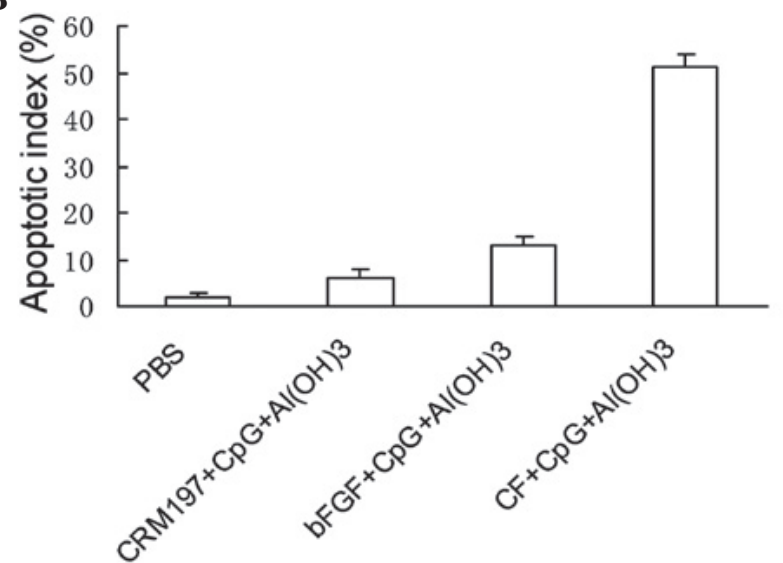

Figure 5. Detection of apoptosis by TUNEL staining of tumor tissues. On day 62 , tumor tissues were sectioned and stained with FITC-dUTP. The percentage of apoptosis was determined by counting the number of apoptotic cells and dividing by the total number of cells in the field. (A) TUNEL staining in each group (original magnification, x200). (B) Percentage of apoptosis in each group.
A

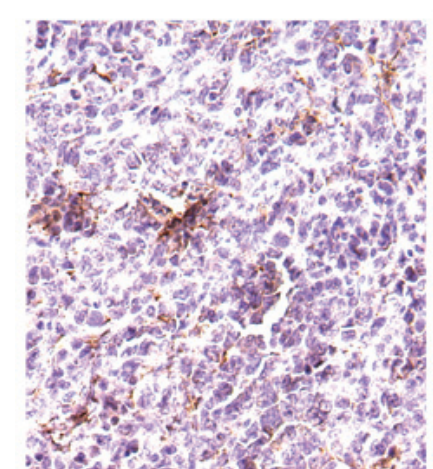
and PBS
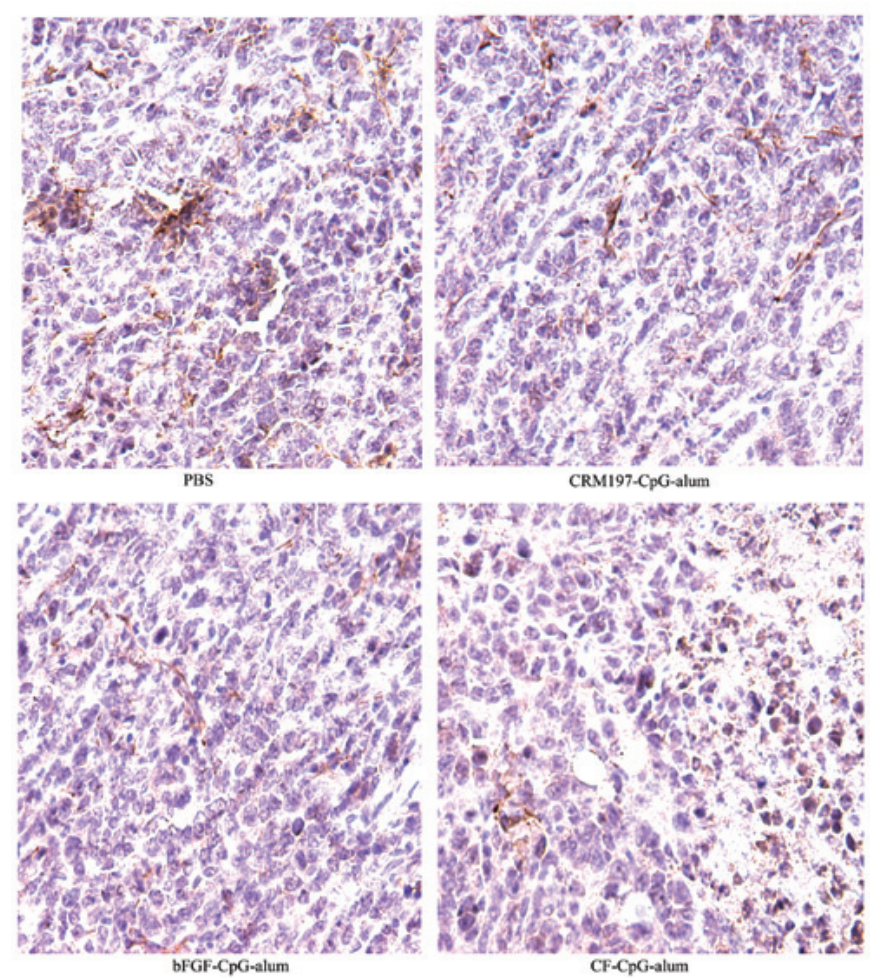

B

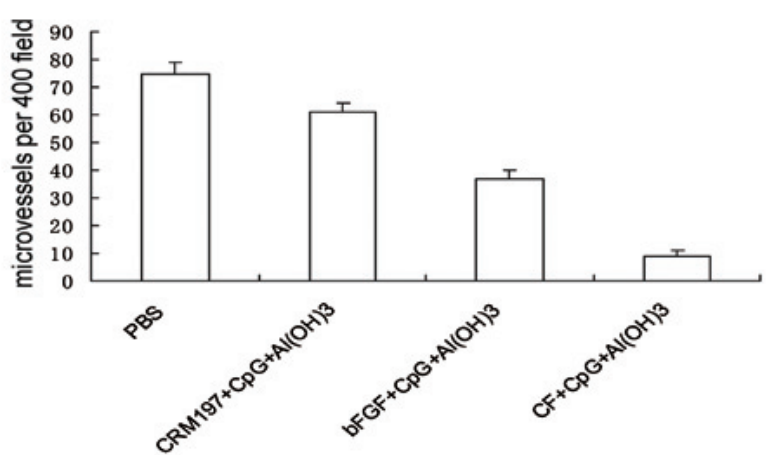

Figure 6. Inhibition of angiogenesis within tumor tissue stained by CD31. On day 62, tumor tissues were sectioned and stained with CD31. Vascular density was estimated by counting the number of microvessels per high power field (magnification, $x 400$ ). (A) CD31 staining of microvessels in each group. (B) Vascular density in each group. 


\section{Discussion}

It has become increasingly clear that a number of diseases including cancer depend on angiogenesis. More than a century ago, it was observed that tumor growth was associated with increased vascularity (33). Based on this, a large number of studies concerning tumor angiogenesis were carried out. In 1945 , it was proposed by Algire et al that 'the rapid growth on tumor transplants is dependent upon the development of a rich vascular supply' (34). Subsequently, Folkman found that tumor tissue could not exceed a size limit of 2-3 $\mathrm{mm}^{3}$ without involving angiogenesis, and that the size was limited by the diffusion of nutrients and gases including $\mathrm{CO}_{2}$ and $\mathrm{O}_{2}$. $\mathrm{He}$ therefore proposed that development of vasculature was critical for tumor growth and that anti-angiogenesis may be an effective strategy for cancer therapy $(3,35)$.

Tumor angiogenesis is a complex process mediated by an 'angiogenic switch'. Angiogenic switch is the transition from the latent to the invasive phase of malignancy. In tumor development, this switch can be mediated by the upregulation of proangiogenitors or the downregulation of antiangiogenitors. The proangiogenitors include the platelet-derived growth factor (PDGF), VEGF, interleukin- 8 and bFGF, whereas the antiangiogenitors include angiostatin and endostatin proteins (36-39). This indicates that tumor growth can be repressed or terminated by inhibiting or neutralizing the angiogenic stimulators (17). In the past decade, the approach has been confirmed by Hori et al and Coppola et al with monoclonal $\mathrm{Ab}$ and receptor antagonists $(40,41)$.

In these processes, investigators have noted that bFGF plays an important role in angiogenesis and tumor progression $(17,19,20)$. The angiogenic activity of bFGF has been demonstrated in the gelatin sponge, the corneal eye pocket, mouse cornea, and subcutaneous Matrigel injection (42-44). Similarly, a low-metastatic renal carcinoma cell line that was transfected with the bFGF gene was capable of expressing bFGF, resulting in an increased angiogenic response in an experimetal animal model (45). Furthermore, elevated serum bFGF levels have been observed in non-small cell lung cancer, head and neck cancer, lymphoma, prostate carcinoma, and soft tissue sarcoma $(15,46)$. Therapy based on bFGF may therefore represent an effective treatment modality for solid tumors.

It has been established that there are approximately five levels on which to inhibit or neutralize the activity of bFGF (47). In the past decade, researchers have utilized various methods to demonstrate this therapy in several tumor models. Hori et al reported that an anti-bFGF monoclonal antibody was capable of inhibiting angiogenesis and tumor growth (41). Schilling-Schon et al revealed that neutralizing anti-FGFR antibodies could block bFGF-mediated angiogenesis in vivo (48). Wang and Becker demonstrated that antisense cDNAs for bFGF and FGFR-1 resulted in inhibition of angiogenesis and tumor growth in nude mice implanted with human melanomas (49). Moreover, Plum et al indicated that the heparin binding domain peptide of bFGF, presenting in a liposome/adjuvant formulation, could effectively inhibit angiogenesis and tumor growth in murine B16BL6 melanoma and Lewis lung carcinoma (17). However, due to its short half-life in vivo, the biological activity of free-form bFGF is limited. It appears to be difficult to sustain therapeutic concentrations in vivo (22).

In addition to this, we have observed through our previous studies that bFGF alone cannot stimulate the body to produce a strong immune response due to its low immunogenicity. In the present study, $\mathrm{CF}$ did not exhibit any proliferation of NIH-3T3 cells in vitro. In vivo, we observed that CF was capable of enhancing immunogenicity with lower concentrations of bFGF and regulating the subclasses and quantities of IgG. Moreover, we observed that $\mathrm{CF}$ together with $\mathrm{CpG}$ and alum as an adjuvant could inhibit tumor growth more effectually than other groups. TUNEL and CD31 staining of were performed for each group to define the anti-tumor mechanism. The results revealed that once tumor angiogenesis was suppressed and the diffusion of nutrients and gases to the tumor was halted, tumor cell apoptosis would occur, and tumor growth would consequently be inhibited.

In summary, until recently, studies of CRM197 as an intramolecular adjuvant to treat tumors have been rarely reported. Our data demonstrate that $\mathrm{CF}$ together with $\mathrm{CpG}$ and alum as an adjuvant enhances the immunogenicity of bFGF and exerts an inhibitory effect on tumor angiogenesis and growth. They have also clarified the immunological mechanism and contribution of this combined vaccine in tumor therapy. Our findings collectively indicate that CRM197 may be an effectual intramolecular adjuvant and that CF may be considered as a new treatment approach for solid tumors.

\section{Acknowledgements}

The study was supported by the Program for New Century Excellent Talents in University (NCET)(NCET-10-0575) and the National Natural Science Foundation of China (Grant no. 31070815).

\section{References}

1. Griffioen AW, Barendsz-Janson AF, Mayo KH and Hillen HF: Angiogenesis, a target for tumor therapy. J Lab Clin Med 132: 363-368, 1998 .

2. Carmeliet P and Jain RK: Angiogenesis in cancer and other diseases. Nature 407: 249-257, 2000.

3. Folkman J: Tumor angiogenesis: therapeutic implications. $\mathrm{N}$ Engl J Med 285: 1182-1186, 1971.

4. Makrilia N, Lappa T, Xyla V, Nikolaidis I and Syrigos K: The role of angiogenesis in solid tumours: an overview. Eur J Intern Med 20: 663-671, 2009.

5. Bhat TA and Singh RP: Tumor angiogenesis - a potential target in cancer chemoprevention. Food Chem Toxicol 46: 1334-1345, 2008.

6. Bremnes RM, Camps C and Sirera R: Angiogenesis in non-small cell lung cancer: the prognostic impact of neoangiogenesis and the cytokines VEGF and bFGF in tumours and blood. Lung Cancer 51: 143-158, 2006.

7. Sridhar SS and Shepherd FA: Targeting angiogenesis: a review of angiogenesis inhibitors in the treatment of lung cancer. Lung Cancer 42 Suppl 1: 81-91, 2003.

8. Kerbel R and Folkman J: Clinical translation of angiogenesis inhibitors. Nat Rev Cancer 2: 727-739, 2002.

9. Risau W: Mechanisms of angiogenesis. Nature 386: 671-674, 1997.

10. Carmeliet P: Mechanisms of angiogenesis and arteriogenesis. Nat Med 6: 389-395, 2000.

11. Greenblatt $M$ and Shubi P: Tumor angiogenesis: transfilter diffusion studies in the hamster by the transparent chamber technique. J Natl Cancer Inst 41: 111-124, 1968.

12. Ehrmann RL and Knoth M: Choriocarcinoma. Transfilter stimulation of vasoproliferation in the hamster cheek pouch Studied by light and electron microscopy. J Natl Cancer Inst 41: 1329-1341, 1968. 
13. Hanahan D and Folkman J: Patterns and emerging mechanisms of the angiogenic switch during tumorigenesis. Cell 86: 353-364, 1996.

14. Folkman J: Angiogenesis and angiogenesis inhibition: an overview. EXS 79: 1-8, 1997

15. Presta M, Dell'Era P, Mitola S, Moroni E, Ronca R and Rusnati M: Fibroblast growth factor/fibroblast growth factor receptor system in angiogenesis. Cytokine Growth Factor Rev 16: $159-178,2005$

16. Beenken A and Mohammadi M: The FGF family: biology, pathophysiology and therapy. Nat Rev Drug Discov 8: 235-253, 2009.

17. Plum SM, Holaday JW, Ruiz A, Madsen JW, Fogler WE and Fortier AH: Administration of a liposomal FGF-2 peptide vaccine leads to abrogation of FGF-2-mediated angiogenesis and tumor development. Vaccine 19: 1294-1303, 2000.

18. Grose R and Dickson C: Fibroblast growth factor signaling in tumorigenesis. Cytokine Growth Factor Rev 16: 179-186, 2005.

19. Javerzat S, Auguste P and Bikfalvi A: The role of fibroblast growth factors in vascular development. Trends Mol Med 8: 483-489, 2002

20. Bikfalvi A, Klein S, Pintucci G and Rifkin DB: Biological roles of fibroblast growth factor-2. Endocr Rev 18: 26-45, 1997.

21. Cronauer MV, Schulz WA, Seifert HH, Ackermann R and Burchardt M: Fibroblast growth factors and their receptors in urological cancers: basic research and clinical implications. Eur Urol 43: 309-319, 2003

22. Liu Y, Sun L, Huan Y, Zhao H and Deng J: Application of bFGF and BDNF to improve angiogenesis and cardiac function. J Surg Res 136: 85-91, 2006.

23. Horvath KA, Doukas J, Lu CY, et al: Myocardial functional recovery after fibroblast growth factor 2 gene therapy as assessed by echocardiography and magnetic resonance imaging. Ann Thorac Surg 74: 481-486; discussion 487, 2002.

24. Miyamoto S, Hirata M, Yamazaki A, et al: Heparin-binding EGF-like growth factor is a promising target for ovarian cancer therapy. Cancer Res 64: 5720-5727, 2004.

25. Yagi H, Yotsumoto F, Sonoda K, Kuroki M, Mekada E and Miyamoto S: Synergistic anti-tumor effect of paclitaxel with CRM197, an inhibitor of HB-EGF, in ovarian cancer. Int Cancer 124: 1429-1439, 2009.

26. Daum RS, Hogerman D, Rennels MB, et al: Infant immunization with pneumococcal CRM197 vaccines: effect of saccharide size on immunogenicity and interactions with simultaneously administered vaccines. J Infect Dis 176: 445-455, 1997.

27. Kamboj KK, King CL, Greenspan NS, Kirchner HL and Schreiber JR: Immunization with Haemophilus influenzae type b-CRM(197) conjugate vaccine elicits a mixed Th1 and Th2 $\mathrm{CD}(4+) \mathrm{T}$ cell cytokine response that correlates with the isotype of antipolysaccharide antibody. J Infect Dis 184: 931-935, 2001.

28. Kamboj KK, Kirchner HL, Kimmel R, Greenspan NS and Schreiber JR: Significant variation in serotype-specific immunogenicity of the seven-valent Streptococcus pneumoniae capsular polysaccharide-CRM197 conjugate vaccine occurs despite vigorous T cell help induced by the carrier protein. J Infect Dis 187: 1629-1638, 2003.

29. McCluskie MJ and Davis HL: CpG DNA is a potent enhancer of systemic and mucosal immune responses against hepatitis B surface antigen with intranasal administration to mice. J Immunol 161: 4463-4466, 1998.

30. Davis HL, Weeratna R, Waldschmidt TJ, Tygrett L, Schorr J and Krieg AM: CpG DNA is a potent enhancer of specific immunity in mice immunized with recombinant hepatitis B surface antigen. J Immunol 160: 870-876, 1998.
31. Chaung HC: $\mathrm{CpG}$ oligodeoxynucleotides as DNA adjuvants in vertebrates and their applications in immunotherapy. Int Immunopharmacol 6: 1586-1596, 2006.

32. Weidner N, Semple JP, Welch WR and Folkman J: Tumor angiogenesis and metastasis - correlation in invasive breast carcinoma. N Engl J Med 324: 1-8, 1991.

33. Ferrara N: VEGF and the quest for tumour angiogenesis factors. Nat Rev Cancer 2: 795-803, 2002.

34. Algire GH, Chalkley HW, Earle WE, et al: Vascular reactions of normal and malignant tissues in vivo. III. Vascular reactions of mice to fibroblasts treated in vitro with methylcholanthrene. J Natl Cancer Inst 11: 555-580, 1950

35. Folkman J, Merler E, Abernathy C and Williams G: Isolation of a tumor factor responsible for angiogenesis. J Exp Med 133: 275-288, 1971

36. Bikfalvi A, Javerzat S, Perollet $\mathrm{C}$ and Savona C: [Angiogenesis and cancer]. Bull Cancer 84: 885-890, 1997.

37. Bouck N, Stellmach V and Hsu SC: How tumors become angiogenic. Adv Cancer Res 69: 135-174, 1996.

38. Liotta LA, Steeg PS and Stetler-Stevenson WG: Cancer metastasis and angiogenesis: an imbalance of positive and negative regulation. Cell 64: 327-336, 1991.

39. Yang LP, Cheng P, Peng XC, et al: Anti-tumor effect of adenovirus-mediated gene transfer of pigment epitheliumderived factor on mouse B16-F10 melanoma. J Exp Clin Cancer Res 28: 75, 2009.

40. Coppola G, Atlas-White M, Katsahambas S, Bertolini J, Hearn MT and Underwood JR: Effect of intraperitoneally, intravenously and intralesionally administered monoclonal anti-beta-FGF antibodies on rat chondrosarcoma tumor vascularization and growth. Anticancer Res 17: 2033-2039, 1997.

41. Hori A, Sasada R, Matsutani E, et al: Suppression of solid tumor growth by immunoneutralizing monoclonal antibody against human basic fibroblast growth factor. Cancer Res 51: 6180-6184, 1991.

42. Watanabe M, McCormick KL, Volker K, et al: Regulation of local host-mediated anti-tumor mechanisms by cytokines: direct and indirect effects on leukocyte recruitment and angiogenesis. Am J Pathol 150: 1869-1880, 1997

43. Wilting J and Christ B: A morphological study of the rabbit corneal assay. Ann Anat 174: 549-556, 1992.

44. Passaniti A, Taylor RM, Pili R, et al: A simple, quantitative method for assessing angiogenesis and antiangiogenic agents using reconstituted basement membrane, heparin, and fibroblast growth factor. Lab Invest 67: 519-528, 1992.

45. Miyake H, Hara I, Yoshimura K, et al: Introduction of basic fibroblast growth factor gene into mouse renal cell carcinoma cell line enhances its metastatic potential. Cancer Res 56: 2440-2445, 1996.

46. Poon RT, Fan ST and Wong J: Clinical implications of circulating angiogenic factors in cancer patients. J Clin Oncol 19: $1207-1225,2001$.

47. Ribatti D, Vacca A, Rusnati M and Presta M: The discovery of basic fibroblast growth factor/fibroblast growth factor-2 and its role in haematological malignancies. Cytokine Growth Factor Rev 18: 327-334, 2007.

48. Schilling-Schon A, Pleyer U, Hartmann C and Rieck PW: The role of endogenous growth factors to support corneal endothelial migration after wounding in vitro. Exp Eye Res 71: 583-589, 2000.

49. Wang Y and Becker D: Antisense targeting of basic fibroblast growth factor and fibroblast growth factor receptor-1 in human melanomas blocks intratumoral angiogenesis and tumor growth. Nat Med 3: 887-893, 1997. 